TITLE:

\title{
Direct determination of second- order density matrix using density equation: Open-shell system and excited state
}

\section{$\operatorname{AUTHOR}(\mathrm{S})$ :}

Nakata, M; Ehara, M; Yasuda, K; Nakatsuji, H

\section{CITATION:}

Nakata, M ... [et al]. Direct determination of second-order density matrix using density equation: Open-shell system and excited state. JOURNAL OF CHEMICAL PHYSICS 2000, 112(20): 8772-8778

ISSUE DATE:

2000-05-22

URL:

http://hdl.handle.net/2433/50178

\section{RIGHT:}

Copyright 2000 American Institute of Physics. This article may be downloaded for personal use only. Any other use requires prior permission of the author and the American Institute of Physics. 


\title{
Direct determination of second-order density matrix using density equation: Open-shell system and excited state
}

\author{
Maho Nakata, Masahiro Ehara, Koji Yasuda, ${ }^{\text {a) }}$ and Hiroshi Nakatsuji ${ }^{\text {b) }}$ \\ Department of Synthetic Chemistry and Biological Chemistry, Faculty of Engineering, Kyoto University, \\ Kyoto 606, Japan
}

(Received 25 August 1999; accepted 1 March 2000)

\begin{abstract}
We formulated the density equation theory (DET) using the spin-dependent density matrix (SDM) as a basic variable and calculated the density matrices of the open-shell systems and excited states, as well as those of the closed-shell systems, without any use of the wave function. We calculated the open-shell systems, $\mathrm{Be}\left({ }^{3} S\right), \mathrm{Be}^{-}\left({ }^{2} S\right), \mathrm{B}^{+}\left({ }^{3} S\right), \mathrm{B}\left({ }^{2} S\right), \mathrm{C}^{2+}\left({ }^{3} S\right), \mathrm{C}^{+}\left({ }^{2} S\right), \mathrm{N}^{3+}\left({ }^{3} S\right)$, and $\mathrm{N}^{2+}\left({ }^{2} S\right)$, and the closed-shell systems, $\mathrm{Be}, \mathrm{Be}^{2-}, \mathrm{B}^{+}, \mathrm{B}^{-}, \mathrm{C}^{2+}, \mathrm{N}^{3+}, \mathrm{H}_{2} \mathrm{O}$, and HF. The new properties calculated are the transition energies and the spin densities at the nuclei. Generally speaking, the accuracy of the present results is slightly worse than that of the previous one using the spin-independent density matrix. ㅇ 2000 American Institute of Physics. [S0021-9606(00)30320-8]
\end{abstract}

\section{INTRODUCTION}

Since all the operators appearing in quantum mechanics are one- and two-body ones, all elemental physical quantities can be determined from the second-order density matrices (2-DMs): The many-electron wave function involves more information than we need to know. Hence, it may be desirable to use the 2-DM as a basic variable of quantum mechanics instead of the wave function. However, a difficulty in this approach is that the $N$-representability condition, which is the condition enforced by the Pauli principle on the DMs, is still not completely known.

One of the authors proposed a nonvariational method for a direct determination of DM in time-independent ${ }^{1}$ and time-dependent ${ }^{2}$ cases. He showed that the density equation (DE) he derived is equivalent to the Schrödinger equation in the domain of $N$-representable DMs. However, the DE contains second-, third- and fourth-order DMs, so that the number of unknown variables exceeds the number of conditions. When the relations between these DMs are given by the $N$-representability condition, ${ }^{2}$ or by some approximate concept, we can directly determine the DM by solving the DE. We call this approach the density equation theory (DET). A review of DET has been summarized recently. ${ }^{3}$

Valdemoro and co-workers ${ }^{4}$ proposed approximate relations for 2-, 3-, and 4-DMs based on the fermion's anticommutation relation. We derived more accurate relations via Green's function method, ${ }^{5,6}$ and successfully determined the 2-DMs of molecules for the first time without any use of the wave function. In terms of electron correlation, Valdemoro's formula correspond to the first-order approximations and ours to the second-order one. Mazziotti gave a reformulation of this approach and some refined approximation., ${ }^{7,8}$ Recently, the DET has further been applied to the calculations of the potential energy curves, equilibrium geometries, and vibrational frequencies of molecules. ${ }^{9}$ Here, we formulate the DET using spin-dependent DMs (SDMs) as basic

\footnotetext{
a) Present address: Graduate School of Human Informatics, Nagoya University, Chikusa-ku, Nagoya 464-01, Japan.

${ }^{b)}$ Author to whom correspondence should be addressed.
}

variables, instead of the spin-independent ones, in order to apply it to open-shell and excited states.

\section{THEORETICAL OUTLINE}

The systems we are interested in are composed of $N$ fermions, whose Hamiltonian involves up to two-body interaction terms,

$$
\hat{H}=\sum_{i} v(i)+\sum_{i>j} w(i, j) .
$$

The matrix form of the Hamiltonian given by

$$
H_{j_{1} j_{2}}^{i_{1} i_{2}}=w_{j_{1} j_{2}}^{i_{1} i_{2}}+\frac{1}{N-1}\left(v_{j_{1}}^{i_{1}} \delta_{j_{2}}^{i_{2}}+v_{j_{2}}^{i_{1}} \delta_{j_{1}}^{i_{2}}\right)
$$

is convenient for the present study. Ensemble density matrix $\rho$ is defined by

$$
\rho=\sum_{m} \alpha_{m} \Psi_{m} \Psi_{m}^{*},
$$

where

$$
\begin{aligned}
& 0 \leqslant \alpha_{m} \leqslant 1, \\
& \sum_{m} \alpha_{m}=1,
\end{aligned}
$$

and $\Psi_{m}$ is an antisymmetric $N$-particle function. $\rho$ describes a pure state when the sum consists of only a single term, i.e.,

$$
\rho=\Psi \Psi^{*} .
$$

The $n$ th-order density matrices ${ }^{(n)} \Gamma$ are defined by

$$
\begin{aligned}
& { }^{(n)} \Gamma\left(x_{1}^{\prime} \cdots x_{n}^{\prime} \mid x_{1} \cdots x_{N}\right) \\
& \quad={ }_{N} C_{n} \int \rho\left(x_{1}^{\prime} \cdots x_{n}^{\prime} x_{n+1} \cdots x_{N} \mid x_{1} \cdots x_{N}\right) d x_{n+1} \cdots d x_{N},
\end{aligned}
$$

where $x_{i}$ stands for the space-spin coordinate of $i$ th electron and ${ }_{N} C_{n}$ the binomial coefficient. Note that we do not integrate the spin variables of the first $n$ particles, so that we are 
able to deal with open-shell system. We refer to ${ }^{(2)} \Gamma$ as $n$-SDM or simply as $n$-DM. Second-quantized definition equivalent to Eq. (2.7) is

$$
{ }^{(n)} \Gamma_{j_{1} j_{2} \cdots j_{n}}^{i_{1} i_{2} \cdots i_{n}}=\sum_{m} \frac{\alpha_{m}}{n !}\left\langle\Psi_{m}\left|a_{i_{1}}^{\dagger} a_{i_{2}}^{\dagger} \cdots a_{i_{n}}^{\dagger} a_{j_{n}} \cdots a_{j_{2}} a_{j_{1}}\right| \Psi_{m}\right\rangle,
$$

where $a^{\dagger}$ and $a$ denote creation and annihilation operators, respectively. The $n$-particle Green's function ${ }^{10}$ is defined as

$$
\begin{aligned}
G^{(n)} & \left(x_{1}^{\prime} t_{1}^{\prime} \cdots x_{n}^{\prime} t_{n}^{\prime} \mid x_{1} t_{1} \cdots x_{n} t_{n}\right) \\
& \left.=(-i)^{n}\left\langle T\left[\phi\left(x_{1}^{\prime} t_{1}^{\prime}\right) \cdots \phi\left(x_{n}^{\prime} t_{n}^{\prime}\right) \phi\left(x_{n} t_{n}\right)^{\dagger} \cdots \phi\left(x_{1} t_{1}\right)^{\dagger}\right)\right]\right\rangle,
\end{aligned}
$$

where $T$ denotes time-ordering operator and $\phi^{\dagger}$ and $\phi$ denote creation and annihilation field operators, respectively. The DMs are related to the Green's function by

$$
\begin{aligned}
& { }^{(n)} \Gamma\left(x_{1}^{\prime} \cdots x_{n}^{\prime} \mid x_{1} \cdots x_{n}\right) \\
& \quad=\frac{(-i)^{n}}{n !} G^{(n)}\left(x_{1}^{\prime} 0^{-} \cdots x_{n}^{\prime} 0^{-} \mid x_{1} 0^{+} \cdots x_{n} 0^{+}\right),
\end{aligned}
$$

where $0^{+}$and $0^{-}$denote positive and negative infinitesimals, respectively.

The $n$ th-order density equation $(\mathrm{DE})^{1}$ is given by

$$
\begin{aligned}
E^{(n)} \Gamma= & \left\{\sum_{i}^{n} v(i)+\sum_{i>j}^{n} w(i, j)\right\}{ }^{(n)} \Gamma+(n+1) \\
& \times \int\left\{v(n+1)+\sum_{i}^{n} w(i, n+1)\right\}^{(n+1)} \Gamma d x_{n+1} \\
& +\frac{1}{2}(n+1)(n+2) \int w(n+1, n+2) \\
& \times^{(n+2)} \Gamma d x_{n+1} d x_{n+2} .
\end{aligned}
$$

In matrix form, it is given by

$$
\begin{aligned}
& E\left\langle\Psi\left|a_{i_{1}}^{\dagger} a_{i_{2}}^{\dagger} \cdots a_{i_{n}}^{\dagger} a_{j_{n}} \cdots a_{j_{2}} a_{j_{1}}\right| \Psi\right\rangle \\
& =\left\langle\Psi\left|\hat{H} a_{i_{1}}^{\dagger} a_{i_{2}}^{\dagger} \cdots a_{i_{n}}^{\dagger} a_{j_{n}} \cdots a_{j_{2}} a_{j_{1}}\right| \Psi\right\rangle .
\end{aligned}
$$

The right hand side of these two equations are the energy density matrix $(\mathrm{EDM}), R^{(n)}$ multiplied by ${ }_{N} C_{n}$. One of the authors proved in 1976 that each DE with $n$ larger than or equal to 2 is equivalent, in necessary and sufficient sense, to the Schrödinger equation if the density matrices involved are $\mathrm{N}$-representable. The matrix form of the second-order DE is written as

$$
\begin{aligned}
E \Gamma_{j_{1} j_{2}}^{i_{1} i_{2}}= & \sum_{j_{3} j_{4} i_{3} i_{4}} H_{j_{3} j_{4}}^{i_{3} i_{4}}\left\langle\Psi\left|a_{i_{1}}^{\dagger} a_{i_{2}}^{\dagger} a_{j_{2}} a_{j_{1}} a_{i_{3}}^{\dagger} a_{i_{4}}^{\dagger} a_{j_{4}} a_{j_{3}}\right| \Psi\right\rangle \\
= & \sum_{j_{3} j_{4}} H_{j_{3} j_{4}}^{j_{1} j_{2}} \Gamma_{i_{1} i_{2}}^{j_{3} j_{4}}+3 \sum_{j_{3} j_{4} i_{4}} H_{j_{3} j_{4}}^{j_{2} i_{4} \Gamma_{i}} \Gamma_{i_{1} i_{2} i_{4}}^{j_{1} j_{3} j_{4}} \\
& +3 \sum_{j_{3} j_{4} i_{3}} H_{j_{3} j_{4}}^{i_{3} j_{1}} \Gamma_{i_{1} i_{2} i_{3}}^{j_{2} j_{3} j_{4}}+6 \sum_{j_{3} j_{4} i_{3} i_{4}} H_{j_{3} j_{4}}^{i_{3} i_{4}} \Gamma_{i_{1} i_{2} i_{3} i_{4}}^{j_{1} j_{2} j_{3} j_{4}} .
\end{aligned}
$$

Our purpose in this paper is to solve this DE. For this purpose, we have to represent approximately the 3,4-DMs included in the EDM in terms of the 1,2-DMs. We use the Green's function method for this purpose in the same way as in the previous paper, ${ }^{5,6}$ but here the DMs explicitly involve the spin variables. The resultant decoupling formula of the $3,4-\mathrm{DMs}$ are written using the wedge product form ${ }^{7}$ as

$$
\begin{aligned}
{ }^{(3)} \Gamma= & { }^{(1)} \Gamma^{3}+3\left({ }^{(2)} \Gamma-{ }^{(1)} \Gamma^{2}\right) \wedge \wedge^{(1)} \Gamma-\sum_{k} P_{k}\left(U_{j_{1} j_{2}}^{k i_{1}} U_{k j_{3}}^{i_{2} i_{3}}+U_{j_{1} j_{2}}^{k i_{2}} U_{k j_{3}}^{i_{3} i_{1}}+U_{j_{1} j_{2}}^{k i_{3}} U_{k j_{3}}^{i_{1} i_{2}}+U_{k i_{1}}^{j_{1} j_{2}} U_{i_{2} i_{3}}^{k j_{3}}+U_{k i_{2}}^{j_{1} j_{2}} U_{i_{3} i_{1}}^{k j_{3}}\right. \\
& +U_{k i_{3}}^{j_{1} j_{2}} U_{i_{1} i_{2}}^{k j_{3}}+U_{j_{2} j_{3}}^{k i_{2}} U_{k j_{1}}^{i_{3} i_{1}}+U_{j_{2} j_{3}}^{k i_{3}} U_{k j_{1}}^{i_{1} i_{2}}+U_{j_{2} j_{3}}^{k i_{1}} U_{k j_{1}}^{i_{2} i_{3}}+U_{k i_{2}}^{j_{2} j_{3}} U_{i_{3} i_{1}}^{k j_{1}}+U_{k i_{3}}^{j_{2} j_{3}} U_{i_{1} i_{2}}^{k j_{1}}+U_{k i_{1}}^{j_{2} j_{3}} U_{i_{2} i_{3}}^{k j_{1}}+U_{j_{3} j_{1}}^{k i_{3}} U_{k j_{2}}^{i_{1} i_{2}} \\
& \left.+U_{j_{3} j_{1}}^{k i_{1}} U_{k j_{2}}^{i_{2} i_{3}}+U_{j_{3} j_{1}}^{k i_{2}} U_{k j_{2}}^{i_{3} i_{1}}+U_{k j_{3}}^{j_{3} j_{1}} U_{i_{1} i_{2}}^{k j_{2}}+U_{k i_{1}}^{j_{3} j_{1}} U_{i_{2} i_{3}}^{k j_{2}}+U_{k i_{2}}^{j_{3} j_{1}} U_{i_{3} i_{1}}^{k j_{2}}\right),
\end{aligned}
$$

$$
\begin{aligned}
{ }^{(4)} \Gamma= & { }^{(1)} \Gamma^{4}+4\left({ }^{(3)} \Gamma-{ }^{(1)} \Gamma^{3}\right) \wedge{ }^{(1)} \Gamma \\
& -6\left({ }^{(2)} \Gamma-{ }^{(1)} \Gamma^{2}\right) \wedge{ }^{(1)} \Gamma^{2}+\frac{3}{4} U \wedge U,
\end{aligned}
$$

where

$$
\Gamma^{n}=\underbrace{\Gamma \wedge \Gamma \cdots \wedge \Gamma}_{n \text { times }} .
$$

$U$ is called collision term and defined by

$$
U=2^{(2)} \Gamma-2\left({ }^{(1)} \Gamma \wedge^{(1)} \Gamma\right) .
$$

$P_{k}$ is zero or unity for $k$ being unoccupied and occupied, respectively. This decoupling approximation is essentially of the second-order in the correlation-correction perturbation. Note that for the 3-DMs, the UV term is not simply written down with the wedge product form and this is not an exact second-order correction, and we examined previously some correction terms. ${ }^{6}$

\section{CALCULATIONAL METHOD}

Our basic variable is the spin-dependent 2-SDM, which has about 16 times larger freedom than the spin-independent 
TABLE I. Results for the triplet states: total energy (in a.u.), correlation energy error (in \%), and rms deviation of the ${ }^{(2)} \Gamma$ calculated by the DET and the wave function method. Active space denotes number of occupied MOs $\times$ virtual MOs and electrons denotes number of $\alpha$ electrons $+\beta$ electrons, respectively.

\begin{tabular}{|c|c|c|c|c|c|c|}
\hline \multirow[b]{2}{*}{ System } & \multirow{2}{*}{$\begin{array}{l}\text { Active space } \\
\text { electrons }\end{array}$} & \multirow[b]{2}{*}{ State } & \multicolumn{4}{|c|}{$\begin{array}{l}\text { Total energy (correlation energy error in \%) } \\
\qquad{ }^{(2)} \Gamma \text { error }^{\mathrm{a}}\end{array}$} \\
\hline & & & DET & $\mathrm{HF}$ & SDCI & FCI \\
\hline $\mathrm{Be}$ & $\begin{array}{l}4 \times 4 \\
3+1\end{array}$ & ${ }^{3} S$ & $\begin{array}{l}-13.31466(-0.2) \\
1.43 \times 10^{-3}\end{array}$ & $\begin{array}{c}-13.30361(100) \\
2.67 \times 10^{-2}\end{array}$ & $\begin{array}{l}-13.31464(0.0) \\
0\end{array}$ & $\begin{array}{c}-13.31464 \\
0\end{array}$ \\
\hline $\mathrm{B}^{+}$ & $\begin{array}{l}4 \times 4 \\
3+1\end{array}$ & ${ }^{3} S$ & $\begin{array}{l}-23.60534(-0.2) \\
\quad 5.21 \times 10^{-3}\end{array}$ & $\begin{array}{c}-23.59233(100) \\
2.66 \times 10^{-2}\end{array}$ & $\begin{array}{l}-23.60532(0.0) \\
0\end{array}$ & $\begin{array}{l}-23.60532 \\
0\end{array}$ \\
\hline $\mathrm{C}^{2+}$ & $\begin{array}{l}4 \times 4 \\
3+1\end{array}$ & ${ }^{3} S$ & $\begin{array}{l}-35.30435(-0.3) \\
\quad 6.10 \times 10^{-3}\end{array}$ & $\begin{array}{c}-35.29153(100) \\
2.14 \times 10^{-2}\end{array}$ & $\begin{array}{l}-35.30431(0.0) \\
0\end{array}$ & $\begin{array}{c}-35.30431 \\
0\end{array}$ \\
\hline $\mathrm{N}^{3+}$ & $\begin{array}{l}4 \times 4 \\
3+1\end{array}$ & ${ }^{3} S$ & $\begin{aligned}-49.36284(-0.2) \\
\quad 4.54 \times 10^{-3}\end{aligned}$ & $\begin{array}{c}-49.34852(100) \\
2.32 \times 10^{-2}\end{array}$ & $\begin{array}{l}-49.36281(0.0) \\
0\end{array}$ & $\begin{array}{l}-49.36281 \\
0\end{array}$ \\
\hline
\end{tabular}

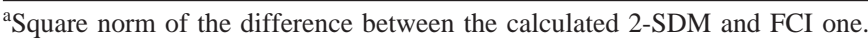

2-DM. It is hermitian and antisymmetric. The 3,4-SDMs are represented in terms of the 1,2-SDMs by Eqs. (2.14) and (2.15). The solution of the DE corresponds to finding the vanishing value of the function, $f$,

$$
f\left({ }^{(2)} \Gamma\right)={ }_{N} C_{2} R\left({ }^{(2)} \Gamma\right)-E^{(2)} \Gamma .
$$

This function is linearized and solved by using the NewtonRaphson method. The algorithm is essentially the same as the previous on $\mathrm{e}^{5,6}$ and is summarized as follows:

(1) Guess initial 2-SDM, which is ordinally Hartree-Fock (HF) ${ }^{(2)} \Gamma$.

(2) Calculate $E=\operatorname{Tr}\left({ }^{(2)} \Gamma H\right)$.

(3) Construct 3,4-SDM ${ }^{(3)} \Gamma$ and ${ }^{(4)} \Gamma$ with ${ }^{(2)} \Gamma$ and ${ }^{(1)} \Gamma$ by Eqs. (2.14) and (2.15).

(4) Calculate the error function $\boldsymbol{f}$ by Eq. (3.1).

(5) Update 2-SDM using the Newton-Raphson method.

(6) Repeat procedures (2)-(5) until convergence.

(7) Check the $N$-representability of the resultant ${ }^{(2)} \Gamma$.

In applying the Newton-Raphson method, ${ }^{11}$ we need to cal- culate the coefficient matrix $A_{i j}=\partial f_{i} / \partial x_{j}$, where $x$ denote the variable ${ }^{(2)} \Gamma$ itself and $i, j$ denote the four indices of ${ }^{(2)} \Gamma$.

As an initial guess of the 2-SDM, we used the HF estimate,

$$
{ }^{(2)} \Gamma_{j_{1} j_{2}}^{i_{1} i_{2}}=\frac{1}{2}\left(\delta_{j_{1}}^{i_{1}} \delta_{j_{2}}^{i_{2}}-\delta_{j_{2}}^{i_{1}} \delta_{j_{1}}^{i_{2}}\right)
$$

where $\delta_{j}^{i}$ is Kronecker's delta, but when the convergency was not good, we used even the full CI(FCI) 2-SDM.

The above procedure was applied to the open-shell atoms, $\mathrm{Be}\left({ }^{3} S\right), \mathrm{Be}^{-}\left({ }^{2} S\right), \mathrm{B}^{+}\left({ }^{3} S\right), \mathrm{B}\left({ }^{2} S\right), \mathrm{C}^{2+}\left({ }^{3} S\right), \mathrm{C}^{+}\left({ }^{2} S\right)$, $\mathrm{N}^{3+}\left({ }^{3} S\right), \mathrm{N}^{2+}\left({ }^{2} S\right)$ and the closed-shell atoms and molecules, $\mathrm{Be}, \mathrm{Be}^{2-}, \mathrm{B}^{+}, \mathrm{B}^{-}, \mathrm{C}^{2+}, \mathrm{N}^{3+}, \mathrm{H}_{2} \mathrm{O}$ and HF. The basis set of $\mathrm{Be}$ is double- $\zeta s$-type $\mathrm{STO}^{12}$ expanded by six GTOs. ${ }^{13}$ For $\mathrm{B}, \mathrm{C}$ and $\mathrm{N}$, double- $\zeta$ s-type GTOs by Huzinaga ${ }^{14}$ and Dunning ${ }^{15}$ were used. For $\mathrm{H}_{2} \mathrm{O}$ and $\mathrm{HF}$, STO-6G basis was used. The geometries of $\mathrm{H}_{2} \mathrm{O}$ and $\mathrm{HF}$ are the experimental ones. ${ }^{16}$

TABLE II. Results for the doublet states: total energy (in a.u.), correlation energy error (in \%), and rms deviation of the ${ }^{(2)} \Gamma$ calculated by the DET and the wave function method. Active space denotes number of

\begin{tabular}{|c|c|c|c|c|c|c|}
\hline \multirow[b]{2}{*}{ System } & \multirow{2}{*}{$\begin{array}{l}\text { Active space } \\
\text { electrons }\end{array}$} & \multirow[b]{2}{*}{ State } & \multicolumn{4}{|c|}{$\begin{array}{l}\text { Total energy (correlation energy error in \%) } \\
{ }_{(2)} \Gamma \text { error }^{\mathrm{a}}\end{array}$} \\
\hline & & & DET & $\mathrm{HF}$ & SDCI & FCI \\
\hline $\mathrm{Be}^{-}$ & $\begin{array}{l}5 \times 3 \\
3+2\end{array}$ & ${ }^{2} S$ & $\begin{array}{c}-13.24020(-) \\
2.20 \times 10^{-3}\end{array}$ & $\begin{array}{l}\cdots \\
\cdots\end{array}$ & $\begin{array}{l}\cdots \\
\cdots\end{array}$ & $\begin{array}{c}-13.24016 \\
0\end{array}$ \\
\hline B & $\begin{array}{l}5 \times 3 \\
3+2\end{array}$ & ${ }^{2} S$ & $\begin{array}{l}-24.11436(-0.3) \\
\quad 6.03 \times 10^{-3}\end{array}$ & $\begin{array}{c}-24.09747(100) \\
2.28 \times 10^{-1}\end{array}$ & $\begin{array}{c}-24.11431(0.0) \\
3.55 \times 10^{-5}\end{array}$ & $\begin{array}{c}-24.11431 \\
0\end{array}$ \\
\hline $\mathrm{C}^{+}$ & $\begin{array}{l}5 \times 3 \\
3+2\end{array}$ & ${ }^{2} S$ & $\begin{aligned}-36.55658(-0.2) \\
\\
3.77 \times 10^{-3}\end{aligned}$ & $\begin{array}{c}-36.54203(100) \\
1.24 \times 10^{-1}\end{array}$ & $\begin{array}{c}-36.55655(0.0) \\
1.56 \times 10^{-5}\end{array}$ & $\begin{array}{l}-36.55655 \\
0\end{array}$ \\
\hline $\mathrm{N}^{2+}$ & $\begin{array}{l}5 \times 3 \\
3+2\end{array}$ & ${ }^{2} S$ & $\begin{array}{l}-51.61476(-0.2) \\
\quad 2.47 \times 10^{-3}\end{array}$ & $\begin{array}{c}-51.60238(100) \\
1.73 \times 10^{-2}\end{array}$ & $\begin{array}{l}-51.61474(0.0) \\
\quad 7.71 \times 10^{-6}\end{array}$ & $\begin{array}{l}-51.61474 \\
0\end{array}$ \\
\hline
\end{tabular}
occupied MOs $\times$ virtual MOs and electrons denotes number of $\alpha$ electrons $+\beta$ electrons, respectively.

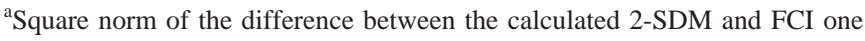


TABLE III. Results for the closed-shell atoms and molecules: total energy (in a.u.), correlation energy error (in $\%)$, and rms deviation of the ${ }^{(2)} \Gamma$ calculated by the DET and the wave function method. Active space denotes number of occupied MOs $\times$ virtual MOs and electrons denotes number of $\alpha$ electrons $+\beta$ electrons, respectively.

\begin{tabular}{|c|c|c|c|c|c|c|}
\hline \multirow[b]{2}{*}{ System } & \multirow{2}{*}{$\begin{array}{l}\text { Active space } \\
\text { electrons }\end{array}$} & \multirow[b]{2}{*}{ State } & \multicolumn{4}{|c|}{$\begin{array}{l}\text { Total energy (correlation energy error in \%) } \\
{ }^{(2)} \Gamma \text { error }^{\mathrm{a}}\end{array}$} \\
\hline & & & DET & $\mathrm{HF}$ & SDCI & FCI \\
\hline $\mathrm{Be}$ & $\begin{array}{l}4 \times 4 \\
2+2\end{array}$ & ${ }^{1} S$ & $\begin{array}{c}-14.58270(-0.1) \\
4.25 \times 10^{-5}\end{array}$ & $\begin{array}{c}-14.56853(100) \\
4.58 \times 10^{-2}\end{array}$ & $\begin{array}{c}-14.58269(0.0) \\
1.06 \times 10^{-4}\end{array}$ & $\begin{array}{l}-14.58269 \\
0\end{array}$ \\
\hline $\mathrm{Be}^{2-}$ & $\begin{array}{l}6 \times 2 \\
3+3\end{array}$ & ${ }^{1} S$ & $\begin{array}{c}-11.26839(0.0) \\
1.32 \times 10^{-5}\end{array}$ & $\begin{array}{c}-11.25896(100) \\
2.00 \times 10^{-2}\end{array}$ & $\begin{array}{l}-11.26839(0.0) \\
0\end{array}$ & $\begin{array}{l}-11.26839 \\
0\end{array}$ \\
\hline $\mathrm{B}^{+}$ & $\begin{array}{l}4 \times 4 \\
2+2\end{array}$ & ${ }^{1} S$ & $\begin{array}{c}-24.24908(1.5) \\
5.19 \times 10^{-5}\end{array}$ & $\begin{array}{c}-24.23434(100) \\
6.03 \times 10^{-2}\end{array}$ & $\begin{array}{c}-24.24929(0.1) \\
5.17 \times 10^{-4}\end{array}$ & $\begin{array}{l}-24.24931 \\
0\end{array}$ \\
\hline $\mathrm{B}^{-}$ & $\begin{array}{l}6 \times 2 \\
2+2\end{array}$ & ${ }^{1} S$ & $\begin{array}{c}-23.66908(-0.2) \\
5.83 \times 10^{-6}\end{array}$ & $\begin{array}{c}-23.65664(100) \\
2.46 \times 10^{-2}\end{array}$ & $\begin{array}{l}-23.66905(0.0) \\
0\end{array}$ & $\begin{array}{l}-23.66905 \\
0\end{array}$ \\
\hline $\mathrm{C}^{2+}$ & $\begin{array}{l}4 \times 4 \\
2+2\end{array}$ & ${ }^{1} S$ & $\begin{array}{c}-36.41774(0.1) \\
1.47 \times 10^{-4}\end{array}$ & $\begin{array}{c}-36.40382(100) \\
3.92 \times 10^{-2}\end{array}$ & $\begin{array}{c}-36.41775(0.1) \\
1.84 \times 10^{-4}\end{array}$ & $\begin{array}{l}-36.41776 \\
0\end{array}$ \\
\hline $\mathrm{N}^{3+}$ & $\begin{array}{l}4 \times 4 \\
2+2\end{array}$ & ${ }^{1} S$ & $\begin{array}{c}-51.08762(-0.1) \\
7.39 \times 10^{-5}\end{array}$ & $\begin{array}{c}-51.07423(100) \\
8.25 \times 10^{-2}\end{array}$ & $\begin{array}{c}-51.08760(0.1) \\
7.96 \times 10^{-5}\end{array}$ & $\begin{array}{l}-51.08761 \\
0\end{array}$ \\
\hline $\mathrm{H}_{2} \mathrm{O}$ & $\begin{array}{l}8 \times 4 \\
4+4\end{array}$ & ${ }^{1} A_{1}$ & $\begin{array}{l}-75.72550(6.9) \\
\quad 9.22 \times 10^{-3}\end{array}$ & $\begin{array}{c}-75.67885(100) \\
2.59 \times 10^{-1}\end{array}$ & $\begin{array}{c}-75.72821(1.5) \\
7.83 \times 10^{-3}\end{array}$ & $\begin{array}{l}-75.72894 \\
0\end{array}$ \\
\hline $\mathrm{HF}$ & $\begin{array}{l}8 \times 2 \\
4+4\end{array}$ & ${ }^{1} \Sigma$ & $\begin{array}{l}-99.52361(8.4) \\
1.32 \times 10^{-5}\end{array}$ & $\begin{array}{l}-99.49984(100) \\
2.00 \times 10^{-2}\end{array}$ & $\begin{array}{l}-99.52577(0.0) \\
0\end{array}$ & $\begin{array}{l}-99.52577 \\
0\end{array}$ \\
\hline
\end{tabular}

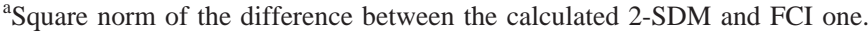

\section{RESULTS}

First, we examine the energy and ${ }^{(2)} \Gamma$ calculated by the present DET. Tables I, II and III show the total energy, the correlation energy error, and the root mean square deviation of the SDMs calculated by the present DET for the openshell triplet, doublet, and the closed-shell singlet states, respectively. Since only $s$-type basis sets are used for atoms, doublet states of the five-electron atoms are not the ground ${ }^{2} P$ state but actually the excited ${ }^{2} S$ states and the triplet states of the four-electron atoms are also not the ${ }^{3} P$ state but the ${ }^{3} S$ state. Computationally, such $S$ states are easier to calculate than the $P$ states. For the triplet states summarized in Table I, the errors in the correlation energy are less than

TABLE IV. Transition energy, ionization energy and electron affinity (in a.u.) calculated by the DET and the wave function method.

\begin{tabular}{lccccr}
\hline \hline System & Transition & DET & HF & \multicolumn{1}{c}{ SDCI } & \multicolumn{1}{c}{ FCI } \\
\hline Be & ${ }^{1} S \rightarrow{ }^{3} S$ & 1.26804 & 1.26492 & 1.26805 & 1.26805 \\
& ${ }^{1} S+e^{-} \rightarrow{ }^{2} S$ & 1.34249 & $\ldots$ & $\cdots$ & 1.34253 \\
& ${ }^{2} S+e^{-} \rightarrow{ }^{1} S$ & 0.44528 & 0.44083 & 0.44526 & 0.44526 \\
& ${ }^{2} S-e^{-} \rightarrow{ }^{1} S$ & -0.13472 & -0.13687 & -0.13498 & -0.13500 \\
$\mathrm{~B}^{+}$ & ${ }^{1} S \rightarrow{ }^{3} S$ & 0.64374 & 0.64201 & 0.64397 & 0.64399 \\
$\mathrm{C}^{2+}$ & ${ }^{1} S \rightarrow{ }^{3} S$ & 1.11339 & 1.11229 & 1.11344 & 1.11345 \\
& ${ }^{1} S+e^{-} \rightarrow{ }^{2} S$ & -0.13884 & -0.13821 & -0.13880 & -0.13879 \\
$\mathrm{~N}^{3+}$ & ${ }^{1} S \rightarrow{ }^{3} S$ & 1.72478 & 1.72571 & 1.72479 & 1.72480 \\
& ${ }^{1} S+e^{-} \rightarrow{ }^{2} S$ & -0.52714 & -0.52815 & -0.52714 & -0.52713 \\
\hline \hline
\end{tabular}

$0.3 \%$ and the total energies of the DET slightly overshoot those of the FCI. Since the DET is not variational, this overshooting happened, though it is small. The rms (root-meansquare) deviation of the SDM is in the order of $10^{-3}$, and is much smaller than the HF ones. For the doublet states shown in Table II, the DET also reproduces well the FCI results almost in the same accuracy as those of the triplet states. For the closed-shell singlet states given in Table III, the energy and ${ }^{(2)} \Gamma$ of the DET show much better agreement with the FCI ones in comparison with the triplet and doublet states. It

TABLE V. $\left\langle N_{\alpha}\right\rangle,\left\langle N_{\beta}\right\rangle,\left\langle S_{z}\right\rangle$ and $\left\langle S^{2}\right\rangle$ calculated for the 2-SDM by the DET

\begin{tabular}{lccccc}
\hline \hline System & State & $\left\langle N_{\alpha}\right\rangle$ & $\left\langle N_{\beta}\right\rangle$ & $\left\langle S_{z}\right\rangle$ & $\left\langle S^{2}\right\rangle$ \\
\hline $\mathrm{Be}$ & ${ }^{3} S$ & 3.00072 & 0.99928 & 1.00072 & 2.00003 \\
$\mathrm{~B}^{+}$ & ${ }^{3} S$ & 2.99741 & 1.00259 & 0.99741 & 2.00237 \\
$\mathrm{C}^{2+}$ & ${ }^{3} S$ & 3.00305 & 0.99694 & 1.00306 & 2.00006 \\
$\mathrm{~N}^{3+}$ & ${ }^{3} S$ & 3.00220 & 0.99780 & 1.00220 & 2.00005 \\
$\mathrm{Be}^{-}$ & ${ }^{2} S$ & 2.99922 & 2.00078 & 0.49922 & 0.75001 \\
$\mathrm{~B}^{+}$ & ${ }^{2} S$ & 3.00161 & 1.99839 & 0.50161 & 0.75000 \\
$\mathrm{C}^{+}$ & ${ }^{2} S$ & 3.00113 & 1.99887 & 0.50113 & 0.75000 \\
& & & & & \\
$\mathrm{Be}^{2}$ & ${ }^{1} S$ & 2.00000 & 2.00000 & 0.00000 & $8.90 \times 10^{-6}$ \\
$\mathrm{Be}^{2-}$ & ${ }^{1} S$ & 3.00000 & 3.00000 & 0.00000 & $4.63 \times 10^{-7}$ \\
$\mathrm{~B}^{+}$ & ${ }^{1} S$ & 2.00004 & 2.00004 & 0.00000 & $4.13 \times 10^{-4}$ \\
$\mathrm{~B}^{-}$ & ${ }^{1} S$ & 3.00000 & 3.00000 & 0.00000 & $-1.94 \times 10^{-6}$ \\
$\mathrm{C}^{2+}$ & ${ }^{1} S$ & 2.00000 & 2.00000 & 0.00000 & $3.87 \times 10^{-5}$ \\
$\mathrm{~N}^{3+}$ & ${ }^{1} S$ & 2.00000 & 2.00000 & 0.00000 & $4.74 \times 10^{-6}$ \\
$\mathrm{H}_{2} \mathrm{O}$ & ${ }^{1} A_{1}$ & 5.00002 & 5.00002 & 0.00000 & $1.28 \times 10^{-2}$ \\
$\mathrm{HF}$ & ${ }^{2} \Sigma$ & 5.00002 & 5.00002 & 0.00000 & $1.03 \times 10^{-2}$ \\
\hline \hline
\end{tabular}


TABLE VI. Minimum eigenvalues of the $P, Q$ and $G$ matrices, numbers of the eigenvalues smaller than $-1.0 \times 10^{-6}$ in the parentheses, and the range of eigenvalues of 1 -SDM calculated by the DET.

\begin{tabular}{|c|c|c|c|c|c|}
\hline System & $\begin{array}{l}\text { Active } \\
\text { electrons }\end{array}$ & $P$-matrix & $Q$-matrix & $G$-matrix & 1-SDM \\
\hline $\operatorname{Be}\left({ }^{3} S\right)$ & 4 & $-6.545 \times 10^{-4}(7)$ & $-6.688 \times 10^{-4}(7)$ & $-7.047 \times 10^{-4}(17)$ & $-3.536 \times 10^{-4} \sim 1.0004$ \\
\hline $\mathrm{B}^{+}\left({ }^{3} S\right)$ & 4 & $-1.815 \times 10^{-4}(4)$ & $-1.896 \times 10^{-4}(3)$ & $-1.937 \times 10^{-4}(11)$ & $3.067 \times 10^{-4} \sim 0.9997$ \\
\hline $\mathrm{C}^{2+}\left({ }^{3} S\right)$ & 4 & $-3.009 \times 10^{-3}(8)$ & $-3.015 \times 10^{-3}(9)$ & $-3.132 \times 10^{-3}(24)$ & $-1.548 \times 10^{-3} \sim 1.0016$ \\
\hline $\mathrm{N}^{3+}\left({ }^{3} S\right)$ & 4 & $-2.237 \times 10^{-3}(7)$ & $-2.242 \times 10^{-3}(7)$ & $-2.235 \times 10^{-5}(23)$ & $1.154 \times 10^{-3} \sim 1.0012$ \\
\hline $\mathrm{Be}^{-}\left({ }^{2} S\right)$ & 5 & $-4.952 \times 10^{-6}(1)$ & $-6.809 \times 10^{-6}(8)$ & $-7.965 \times 10^{-6}(6)$ & $2.659 \times 10^{-4} \sim 1.0000$ \\
\hline $\mathrm{B}\left({ }^{2} S\right)$ & 5 & $-2.123 \times 10^{-3}(6)$ & $-2.121 \times 10^{-4}(10)$ & $-4.233 \times 10^{-4}(23)$ & $-2.102 \times 10^{-4} \sim 1.0021$ \\
\hline $\mathrm{C}^{+}\left({ }^{2} S\right)$ & 5 & $-1.331 \times 10^{-3}(5)$ & $-3.015 \times 10^{-3}(7)$ & $-3.132 \times 10^{-3}(18)$ & $-1.316 \times 10^{-3} \sim 1.0000$ \\
\hline $\mathrm{N}^{2+}\left({ }^{2} S\right)$ & 5 & $-1.555 \times 10^{-5}(2)$ & $-1.396 \times 10^{-4}(10)$ & $-2.928 \times 10^{-4}(14)$ & $-1.154 \times 10^{-3} \sim 1.0012$ \\
\hline $\operatorname{Be}\left({ }^{1} S\right)$ & 4 & $-6.246 \times 10^{-6}(1)$ & $-3.938 \times 10^{-6}(2)$ & $-4.781 \times 10^{-6}(4)$ & $1.119 \times 10^{-4} \sim 0$. \\
\hline $\mathrm{Be}^{2-}\left({ }^{1} S\right)$ & 6 & ok & $-3.045 \times 10^{-6}(5)$ & $-4.576 \times 10^{-6}(6)$ & $1.996 \times 10^{-4} \sim 1.0000$ \\
\hline $\mathrm{B}^{+}\left({ }^{1} S\right)$ & 4 & $-6.963 \times 10^{-6}(4)$ & $-1.563 \times 10^{-5}(5)$ & $-4.576 \times 10^{-6}(12)$ & $1.996 \times 10^{-4} \sim 1.0000$ \\
\hline $\mathrm{B}^{-}\left({ }^{1} S\right)$ & 6 & ok & $-1.417 \times 10^{-4}(4)$ & $-1.986 \times 10^{-4}(6)$ & $3.009 \times 10^{-4} \sim 1.0000$ \\
\hline $\mathrm{C}^{2+}\left({ }^{1} S\right)$ & 4 & $-3.818 \times 10^{-5}(5)$ & $-2.019 \times 10^{-6}(1)$ & $-1.998 \times 10^{-5}(7)$ & $5.863 \times 10^{-4} \sim 0.9998$ \\
\hline $\mathrm{N}^{3+}\left({ }^{1} S\right)$ & 4 & $-1.922 \times 10^{-5}(2)$ & ok & $-1.111 \times 10^{-5}(7)$ & $1.168 \times 10^{-1} \sim 0.9999$ \\
\hline $\mathrm{H}_{2} \mathrm{O}\left({ }^{1} A_{1}\right)$ & 8 & $-6.793 \times 10^{-4}(2)$ & $-4.830 \times 10^{-4}(18)$ & $-6.606 \times 10^{-4}(5)$ & $1.427 \times 10^{-2} \sim 0.9987$ \\
\hline $\operatorname{HF}\left({ }^{1} \Sigma\right)$ & 8 & $-8.303 \times 10^{-4}(1)$ & $-7.688 \times 10^{-4}(14)$ & $-1.221 \times 10^{-3}(5)$ & $1.696 \times 10^{-2} \sim 0.9994$ \\
\hline
\end{tabular}

should be noted that the ${ }^{(2)} \Gamma$ by the DET is more accurate than those of the SDCI, since the DET determines the DM directly.

The transition energy, ionization energy, and electron affinity are summarized in Table IV for $\mathrm{Be}, \mathrm{B}, \mathrm{C}$, and $\mathrm{N}$ atoms. These quantities are calculated for the first time by the DET. Since some states involved are not the normal ground and excited states, the values themselves may look strange, but the DET results are very close to the FCI ones. The deviations of the DET values from the FCI ones are less than $2.8 \times 10^{-4}$ a.u., while those of the HF and SDCI methods are $4.42 \times 10^{-3}$ and $2 \times 10^{-5}$, respectively.

The expectation values of the numbers of $\alpha$ and $\beta$ spin electrons, $\left\langle N_{\alpha}\right\rangle$ and $\left\langle N_{\beta}\right\rangle$, and those of the operators $S_{z}$ and $S^{2},\left\langle S_{z}\right\rangle$ and $\left\langle S^{2}\right\rangle$, are calculated and summarized in Table V for the triplet, doublet, and singlet states. For the closed-shell singlet state, we obtain almost correct expectation values for atoms, but the deviations are somewhat large for molecules, especially for $\left\langle S^{2}\right\rangle$. For the open-shell triplet and doublet states, the expectation values $\left\langle N_{\alpha}\right\rangle,\left\langle N_{\beta}\right\rangle,\left\langle S_{z}\right\rangle$ and $\left\langle S^{2}\right\rangle$ slightly deviate from the exact values. This is because the present approximate decoupling technique does not include any restrictive conditions for the numbers of the electrons and spins. When we enforce the "normalization'" condition, the calculations were not improved and even did not converge.

Next, we examine the $N$-representability conditions for the 1-SDM and 2-SDM. Table VI gives the occupation numbers of the natural orbitals of the 1-SDM, and the lowest eigen values of the $P, Q$ and $G$ matrices. ${ }^{17}$ For the closedshell systems, the $N$-representability condition of the 1-SDM is completely satisfied, i.e., the occupation numbers are all positive and less than unity. This was also so in the previous spin-free calculations. ${ }^{5,6}$ However, the violation of the $N$-representability of the 1-SDM occurs for some open-shell atoms. The $P, Q$ and $G$ are necessary conditions for the $N$-representability of the 2-SDM: The eigenvalues of the $P$, $Q$ and $G$ matrices should be nonnegative. However, some of the eigenvalues are slightly negative, though the violations seem to be small. The extent of the violation seems to increase as the number of the electrons increases, and seems to be larger for the open-shell systems than for the closed-shell systems.

Table VII shows the second moment of electron distribution, $\left\langle r^{2}\right\rangle$. Again, the DET results for the closed-shell systems are better than those for the open-shell systems. Some of them are better than the SDCI results, because the DET directly determines the SDM. For the open-shell systems, the SDCI results are superior to the DET ones, because the violation of the $N$-representability condition tends to accumulate electrons near the nucleus. Some of the SDCI results are identical to the FCI one: as for the active space, it is small, the single and double excitations span the complete space.

Finally, the spin density and the electron density at the nucleus are calculated. In Tables VIII, IX and X, the results are shown for the triplet, doublet, and singlet states, respec-

TABLE VII. Second moment $\left(\left\langle r^{2}\right\rangle\right)$ of atoms calculated by the DET and the wave function method.

\begin{tabular}{lccccc}
\hline \hline System & State & DET & HF & SDCI & FCI \\
\hline $\mathrm{Be}$ & ${ }^{3} S$ & -4.85006 & -4.85021 & -4.85006 & -4.85006 \\
$\mathrm{~B}^{+}$ & ${ }^{3} S$ & -4.95063 & -4.95074 & -4.95070 & -4.95070 \\
$\mathrm{C}^{2+}$ & ${ }^{3} S$ & -3.27401 & -3.27404 & -3.27402 & -3.27402 \\
$\mathrm{~N}^{3+}$ & ${ }^{3} S$ & -2.33906 & -2.33908 & -2.33906 & -2.33906 \\
$\mathrm{Be}^{-}$ & ${ }^{2} S$ & -7.33125 & $\ldots$ & $\ldots$ & -7.33128 \\
$\mathrm{~B}^{+}$ & ${ }^{2} S$ & -6.45884 & -6.45765 & -6.45889 & -6.45892 \\
$\mathrm{C}^{+}$ & ${ }^{2} S$ & -4.08158 & -4.08119 & -4.08159 & -4.08160 \\
$\mathrm{~N}^{2+}$ & ${ }^{2} S$ & -2.84906 & -2.84892 & -2.84907 & -2.84907 \\
$\mathrm{Be}^{2-}$ & ${ }^{1} S$ & -5.77125 & -5.77782 & -5.77136 & -5.77127 \\
$\mathrm{Be}^{2-}$ & ${ }^{1} S$ & -9.56353 & -9.56389 & -9.56355 & -9.56355 \\
$\mathrm{~B}^{+}$ & ${ }^{1} S$ & -2.71924 & -2.69894 & -2.71849 & -2.71887 \\
$\mathrm{~B}^{-}$ & ${ }^{1} S$ & -9.81236 & -9.81253 & -9.81237 & -9.81237 \\
$\mathrm{C}^{2+}$ & ${ }^{1} S$ & -1.56149 & -1.55544 & -1.56144 & -1.56152 \\
$\mathrm{~N}^{3+}$ & ${ }^{1} S$ & -1.02144 & -1.01932 & -1.02143 & -1.02145 \\
\hline \hline
\end{tabular}


TABLE VIII. Electron density and spin density at the nucleus of the triplet state. $\rho$ denotes the total electron density, and $\rho_{\alpha}$ and $\rho_{\beta}$ are the $\alpha$ and $\beta$ electron densities, respectively, and $\Delta \rho=\rho_{\alpha}-\rho_{\beta}$ is spin density at the nucleus.

\begin{tabular}{lrrrr}
\hline \hline System (state) & \multicolumn{1}{c}{ DET } & \multicolumn{1}{c}{ HF } & SDCI & \multicolumn{1}{c}{ FCI } \\
\hline $\operatorname{Be}\left({ }^{3} S\right)$ & & & & \\
$\rho$ & 32.530 & 32.401 & 32.530 & 32.530 \\
$\rho_{\alpha}$ & 18.069 & 17.593 & 18.068 & 18.068 \\
$\rho_{\beta}$ & 14.461 & 14.808 & 14.462 & 14.462 \\
$\Delta \rho$ & 3.608 & 2.785 & 3.605 & 3.605 \\
$\mathrm{~B}^{+}\left({ }^{3} S\right)$ & & & & \\
$\rho$ & 68.741 & 68.680 & 68.742 & 68.742 \\
$\rho_{\alpha}$ & 36.216 & 35.947 & 36.225 & 36.225 \\
$\rho_{\beta}$ & 32.525 & 32.733 & 32.517 & 32.517 \\
$\Delta \rho$ & 3.691 & 3.215 & 3.708 & 3.708 \\
$\mathrm{C}^{2+}\left({ }^{3} S\right)$ & & & & \\
$\rho$ & 122.143 & 122.064 & 122.143 & 122.143 \\
$\rho_{\alpha}$ & 64.502 & 64.083 & 64.489 & 64.489 \\
$\rho_{\beta}$ & 57.641 & 57.981 & 57.654 & 57.654 \\
$\Delta \rho$ & 6.860 & 6.103 & 6.836 & 6.836 \\
$\mathrm{~N}^{3+}\left({ }^{3} S\right)$ & & & & \\
$\rho$ & & & & \\
$\rho_{\alpha}$ & 197.798 & 197.705 & 197.798 & 197.798 \\
$\rho_{\beta}$ & 104.557 & 103.989 & 104.543 & 104.543 \\
$\Delta \rho$ & 93.241 & 93.716 & 93.255 & 93.255 \\
\hline \hline
\end{tabular}

tively. The spin density at the nucleus is a very important observable in ESR and other magnetic chemistry and calculated for the first time by the DET. The DET well reproduces the spin density at the nucleus of the FCI method. The results are almost in the same accuracy as the SDCI ones and much better than the HF ones, since the DET includes both electron and spin correlations up to second-order in the perturbation. The spin densities calculated within the HF method do not include the spin polarization effects ${ }^{18,19}$ and therefore

TABLE IX. Electron density and spin density at the nucleus of the doublet state. $\rho$ is the total electron density, and $\rho_{\alpha}$ and $\rho_{\beta}$ are the $\alpha$ and $\beta$ electron densities, respectively, and $\Delta \rho=\rho_{\alpha}-\rho_{\beta}$ is the spin density at the nucleus.

\begin{tabular}{lrrrr}
\hline \hline System (state) & \multicolumn{1}{c}{ DET } & HF & SDCI & \multicolumn{1}{c}{ FCI } \\
\hline $\mathrm{Be}^{-}\left({ }^{2} S\right)$ & & & & \\
$\rho$ & 33.568 & $\ldots$ & $\ldots$ & 33.568 \\
$\rho_{\alpha}$ & 17.909 & $\ldots$ & $\ldots$ & 17.911 \\
$\rho_{\beta}$ & 15.659 & $\ldots$ & $\ldots$ & 15.658 \\
$\Delta \rho$ & 2.249 & $\ldots$ & $\ldots$ & 2.253 \\
$\mathrm{~B}\left({ }^{2} S\right)$ & & & & \\
$\rho$ & 70.058 & 69.998 & 70.058 & 70.058 \\
$\rho_{\alpha}$ & 35.980 & 35.796 & 35.977 & 35.977 \\
$\rho_{\beta}$ & 34.078 & 34.202 & 34.081 & 34.081 \\
$\Delta \rho$ & 1.902 & 1.594 & 1.896 & 1.896 \\
$\mathrm{C}^{+}\left({ }^{2} S\right)$ & & & & \\
$\rho$ & 125.313 & 125.238 & 125.313 & 125.313 \\
$\rho_{\alpha}$ & 64.033 & 63.809 & 64.030 & 64.030 \\
$\rho_{\beta}$ & 61.280 & 61.429 & 61.284 & 61.283 \\
$\Delta \rho$ & 2.753 & 2.380 & 2.746 & 2.746 \\
$\mathrm{~N}^{2+}\left({ }^{2} S\right)$ & & & & \\
$\rho$ & & & & \\
$\rho_{\alpha}$ & 203.820 & 203.732 & 203.820 & 203.820 \\
$\rho_{\beta}$ & 103.832 & 103.564 & 103.828 & 103.828 \\
$\Delta \rho$ & 99.989 & 100.168 & 99.992 & 99.992 \\
\hline \hline & 3.843 & 3.396 & 3.836 & 3.836 \\
\hline \hline
\end{tabular}

TABLE X. Electron density at the nucleus of the singlet state.

\begin{tabular}{ccrrr}
\hline \hline System $($ state $)$ & DET & HF & \multicolumn{1}{c}{ SDCI } & \multicolumn{1}{c}{ FCI } \\
\hline $\operatorname{Be}^{1}\left({ }^{1} S\right)$ & 31.489 & 31.352 & 31.489 & 31.489 \\
$\mathrm{Be}^{2-}\left({ }^{1} S\right)$ & 34.316 & 34.209 & 34.316 & 34.316 \\
$\mathrm{~B}^{+}\left({ }^{1} S\right)$ & 69.030 & 68.983 & 69.031 & 69.031 \\
$\mathrm{~B}^{-}\left({ }^{1} S\right)$ & 71.456 & 71.399 & 71.457 & 71.457 \\
$\mathrm{C}^{2+}\left({ }^{1} S\right)$ & 123.532 & 123.470 & 123.532 & 123.532 \\
$\mathrm{~N}^{3+}\left({ }^{1} S\right)$ & 201.055 & 200.978 & 201.055 & 201.055 \\
\hline \hline
\end{tabular}

are always smaller than the DET and FCI values. For the closed-shell singlet states, the spin density is exactly zero, therefore, only the total density of the electrons at nucleus is given. The accuracy of the DET is almost the same as that of SDCI.

\section{CONCLUSION}

We successfully calculated the SDMs of some openshell and excited states for the first time by the spin-explicit DET without any use of the wave function. The same method is also applied to the closed-shell systems, though the solution is easier with the spin-free formalism. Generally speaking, the quality of solutions were better for the closedshell systems than for the open-shell systems. A reason is a larger number of variables to be solved for open-shell systems. As seen from the results, the present method and the solution algorithm are not yet complete and needs some future refinement. Nonetheless, the present results constitutes a milestone in the DET approach in theoretical chemistry as its first application to open-shell and excited states.

\section{ACKNOWLEDGMENT}

This study has been supported by the Grand-in-Aid for Scientific Research from the Japanese Ministry of Education, Science, Culture, and Sports.

${ }^{1}$ H. Nakatsuji, Phys. Rev. A 14, 41 (1976).

${ }^{2}$ H. Nakatsuji, Theor. Chem. Acc. 102, 97 (1999).

${ }^{3}$ H. Nakatsuji, Many-Electron Densities and Reduced Density Matrices, edited by J. Cioslowski (Kluwer, New York, 2000).

${ }^{4}$ C. Valdemoro, Phys. Rev. A 45, 4462 (1992); 47, 971 (1993); 47, 979 (1993).

${ }^{5}$ H. Nakatsuji and K. Yasuda, Phys. Rev. Lett. 76, 1039 (1996).

${ }^{6}$ K. Yasuda and H. Nakatsuji, Phys. Rev. A 56, 2648 (1997).

${ }^{7}$ D. A. Mazziotti, Phys. Rev. A 57, 4219 (1998).

${ }^{8}$ D. A. Mazziotti, Phys. Rev. A 60, 4396 (1999).

${ }^{9}$ M. Ehara, M. Nakata, H. Kou, K. Yasuda, and H. Nakatsuji, Chem. Phys. Lett. 305, 483 (1999).

${ }^{10}$ A. L. Fetter and J. D. Walecka, Quantum Theory of Many Particle Systems (McGraw Hill, New York, 1971); A. A. Abrikosov, L. P. Gorkov, and E. Dzyloshinskii, Method of Quantum Field Theory in Statistical Physics (Prentice-Hall, Englewood, Cliffs, NJ, 1963).

${ }^{11}$ W. H. Press, S. A. Teukolsky, W. T. Vetterling, and B. Flannery, Numerical Recipes in C (Cambridge University Press, Cambridge, MA).

${ }^{12}$ E. Clementi and C. Roetti, At. Data Nucl. Data Tables 14, 428 (1974); the exponents were reoptimized by T. Koga and S. Watanabe (private communication).

${ }^{13}$ W. J. Hehre, R. F. Stewart, and J. A. Pople, J. Chem. Phys. 51, 2657 (1969).

${ }^{14}$ S. Huzinaga, J. Chem. Phys. 42, 1293 (1965).

${ }^{15}$ T. H. Dunning, Jr., J. Chem. Phys. 53, 2823 (1970); T. H. Dunning, Jr. and P. J. Hay, in Method of Electronic Structure Theory, Vol. 2, edited by H. F. Schaefer III (Plenum, New York, 1977); Actually, basis sets are 
taken from EMSL Gaussian Basis Set Order Form, http:// www.emsl.pnl.gov:2080/forms/basisform.html.

${ }^{16}$ L. E. Sutton, D. G. Jenkin, and A. D. Mitchell, Tables of Interatomic Distances and Configuration in Molecules and Ions I (The Chemical Society, London, 1958).

${ }^{17}$ C. Garrod, M. V. Mihailovic, and M. Rosina, J. Math. Phys. 16, 868
(1975); A. J. Coleman, Rev. Mod. Phys. 35, 668 (1963); C. Garrod and J. Percus, J. Math. Phys. 5, 1756 (1964); H. Kummer, ibid. 8, 2063 (1967); T. L. Gilbert, Phys. Rev. B 12, 2111 (1975).

${ }^{18}$ T. Yonezawa, H. Nakatsuji, T. Kawamura, and H. Kato, Chem. Phys. Lett. 2, 454 (1968).

${ }^{19}$ H. Nakatsuji, H. Kato, and T. Yonezawa, J. Chem. Phys. 62, 3175 (1969). 